\title{
Codificación y análisis diferencial de los problemas de los adolescentes acogidos en centros de protección en España*
}

\section{Coding and differential analysis of the problems of teenagers residing in protection centers in Spain}

Recibido: octubre 14 de 2009 ～Revisado: febrero 5 de 2010 | Aceptado: marzo 2 de 2010

\author{
JuAn MANUEl FernándeZ-Millán ** \\ Universidad de Granada, España \\ Mª José Malpica Buitrago \\ Universidad de Málaga, España \\ Marina Fernández NaVAS \\ Centro Asistencial de Melilla, España
}

Para citar este artículo. Fernández-Millán, J.M., Malpica Buitrago, M.J. \& Fernández Navas, M. (2010). Codificación y análisis diferencial de los problemas de los adolescentes acogidos en centros de protección en España. Universitas Psychologica, 9 (3), 841-848.

\footnotetext{
* Artículo de investigación.

** C/ Poeta Salvador Rueda, no 11 3ํ A. 52006 Melilla. España. E-mail: fmillan@cop.es
}

\section{RES UMEN}

La adolescencia es una etapa de la vida que se caracteriza por un marcado y difícil desarrollo físico, psicológico y social, durante el que el joven deberá enfrentarse a distintos problemas cotidianos y estresores vitales. Conocer y clasificar los conflictos que más preocupan al adolescente puede servir para dirigir los proyectos educativos o las intervenciones terapéuticas más eficaces. En el presente trabajo, se han codificado y cuantificado, a través del Sistema de Codificación y Análisis Diferencial de los Problemas de los Adolescente, los problemas percibidos como más estresantes por una muestra de menores residentes en un centro de acogida. Una comparación entre los adolescentes acogidos y aquellos que viven con sus padres, muestra que los menores acogidos (entre 11 y 17 años) plantean situaciones vitales diferentes en todas las categorías, destacando el alto porcentaje de problemas interpersonales con compañeros y relacionados con el deporte o el tiempo de ocio.

Palabras clave autores

Adolescencia, conflictos, percepción de problemas, estresores.

\begin{abstract}
A B S T R A C T
Adolescence is a stage of life characterized by a marked and dififficult psysical, psychological and social development in which the young must face different daily problems and vital stressors. Knowing and classifying the conflicts that most worry the teenager might be used to direct the educative projects or therapeutic intervention more efficients. In this paper, problems perceived as more stressing have been codified and quantified through Adolescent's Problems Differential Analysis and Coding System using a sample of minors leaving in a residential child care. A comparison between housed teenagers and those who live with their parents show that housed minors (between 11 and 17 years old) rise different vital situations in every single category. An outstanding fact is the high percentage of interpersonal problems with other house mates and related to sports and leisure time. Keywords authors Adolescence, Conflicts, Problem Perception, Stressors.
\end{abstract}




\section{Introducción}

La adolescencia, como etapa evolutiva, ha recibido una intensa atención por parte de la Psicología, desde sus comienzos; así, y a modo de ejemplo, pueden citarse la obra Adolescence que ya en 1904 escribió Stanley Hall o los estudios de Piaget sobre esta etapa.

Se entiende como adolescencia el periodo de desarrollo físico, psicológico y social que experimenta el sujeto entre los 11 y 21 años, aproximadamente, aunque se suele distinguir entre primera adolescencia o pubertad, segunda adolescencia o adolescencia temprana o media, y tercera adolescencia, adolescencia tardía o edad juvenil (Castillo, 1999).

Muchas son las formas de conceptuar esta etapa, pudiéndose encontrar diferentes matices según la cultura o la época en la que se circunscribe, pero todas estas concepciones, sean de la índole que sean, coinciden en considerar a la adolescencia como periodo inequívoco de cambios y retos, que el adolescente debe afrontar y superar como parte esencial de la vida del individuo y que culminan en un desafío global: hacerse mayor (Castillo, 1999; Gómez-Fraguela, Luengo-Martín, RomeroTriñanes, Villar-Torres, Sobral-Fernández, 2006; Krauskopf, 1995).

La adolescencia puede verse influida o afectada por características personales o situacionales de los jóvenes. Una de las características situacionales, que vamos a destacar en este trabajo, es el hecho de que los adolescentes se encuentran en un centro de acogida debido a características personales o familiares (menores inmigrantes, menores de la calle, menores con grave problemática personal...).

Ante esta realidad, el adolescente presenta una crisis global que atañe a todas las facetas de su vida personal, y que es considerada como elemento esencial de maduración individual, como trampolín necesario para que el niño, que hasta ese momento teníamos ante nuestros ojos, deje de serlo. En todo este proceso, el joven debe verse acompañado por una serie de personas adultas que lo guíen en este camino no exento de dificultades. Por esta razón, si bien los adultos deben establecer fronteras y límites con la intención de inculcar al menor una serie de normas necesarias, también por otro lado, deben apoyarlo en esta etapa a la vez tan rica y complicada, cargada de problemas y decisiones por tomar, que pueden marcar el resto de sus vidas.

Al definirse como etapa de transición entre la infancia y la edad adulta, la adolescencia viene inseparablemente unida a términos como: crisis, reto (Castillo, 1999), inestabilidad y provisionalidad (Garaigordobil, 2000), transición, itinerario y proceso (Funes, 2003) o reorganización (Ochaíta, 1995), llegándose a afirmar que se trata de una "etapa de inmadurez en busca de la madurez" (Castillo, 1999, p. 42) o como "alguien que está aprendiendo a gestionar los riesgos" (Funes, 2003, p. 2).

Con estas características, no es extraño que gran número de los trabajos realizados se hayan centrado en la conflictividad asociada a las relaciones interpersonales del adolescente (Espada, Méndez, Griffin \& Botvin, 2003; Fernández-Millán \& Buela-Casal, 2007; Maciá, 1994; Urra, 1995, 2006; Zavala, Valadez \& Vargas, 2008), hasta el punto de que Funes, en un trabajo de título muy sugerente, "¿Cómo trabajar con adolescentes sin empezar por considerarlos un problema?”, advierte sobre la necesidad de "leer siempre en primer lugar sus conductas en clave adolescente" (Funes, 2003, p. 3). Costa y Morales (1998) realizaron un ensayo en el que se presentan las variables (factores de riesgo) que van a propiciar la aparición de conductas violentas en el niño y en el adolescente, dividiendo los factores de forma sistémica, según de dónde procedan las variables. Por su parte, aquellas investigaciones que se han centrado en los conflictos entre padres e hijos (del Barrio, 1995) afirman que estas disputas tienen que ver con aspectos cotidianos (TV, ayuda doméstica, horas de llegar a casa, tareas escolares e indumentaria). La influencia que todos estos conflictos van a ejercer sobre el adolescente, va a depender de la percepción que éste tenga sobre los mismos.

Ante este estado de cosas, es necesario contar con métodos que nos permitan conocer, desde la perspectiva del propio adolescente, cuáles son los acontecimientos o incidentes que más les preocu- 
pan. A este respecto, diversos autores han intentado dar respuesta a este interrogante, utilizando diversos métodos (Brown, O’Keeffe, Sanders \& Baker, 1986; Coddington, 1972; Kirchner \& Forns, 2000; Moos, 1993). Por su parte, Forns, Amador, Kirchner, Martorell, Zanini y Muro (2004) proponen el Sistema de Codificación y Análisis Diferencial de los Problemas de los Adolescentes. Este sistema posibilita el análisis de los problemas de los jóvenes, libremente expresados por ellos mismos, lo que permite captar la percepción subjetiva que los adolescentes poseen de sus propias dificultades y retos, en el devenir de su día a día. El sistema distingue cuatro categorías: 1) Naturaleza del Problema $(\mathrm{N})$ : identifica a quién le sucede el problema, pudiéndose elegir entre personales (N1), interpersonales (N2) o ajenos (N3); 2) Contenido (C): hace referencia a las características configurativas del problema, al tema, y distingue entre problemas de relación $(\mathrm{C} 1)$, problemas de separación (C2), autoculpa (C3.), rendimiento escolar (C4), cambio de hogar, residencia o escuela (C5), conflicto de normas y reglas (C6), victimización (C7), deporte, ocio o vacaciones (C8), salud, enfermedad o accidente (C9), adicciones (C10), dinero, economía y trabajo (C11), muerte y suicidio (C12) y otros (C13); 3) Participantes (P): se refiere a los intervinientes en el conflicto y está dividida en 8 grupos: el propio sujeto (P1), el núcleo familiar (P2), la familia extensa (P3), los compañeros y amigos (P4), la pareja (P5), los profesores (P6), los adultos (P7) y los animales (P8); y, 4) Ámbito de Ocurrencia $(\mathrm{AO})$ : se centra en el lugar donde se desarrolla la situación conflictiva, que puede ser el hogar y entorno familiar extenso (AO1), escuela (AO2), ocio y deporte (AO3), barrio y contexto laboral $(\mathrm{AO} 4)$ y no especificado (AO5).

En el estudio de estos autores, la aplicación de este sistema se llevó a cabo con 1362 adolescentes de entre 12 y 16 años que vivían con sus familias. Sin embargo, en nuestra investigación, pretendemos implementar este sistema a la población de niños y adolescentes residentes en un centro de acogida, con el objetivo de establecer un análisis comparativo, a fin de concluir si existen diferencias cualitativas entre los problemas que preocupan a los jóvenes en situación de desamparo e "institucionalizados" y los que viven en el seno de un a familia (entendida ésta en su acepción más amplia). Se espera encontrar que los menores acogidos presenten mayor preocupación en problemas personales que los menores que forman parte de un núcleo familiar; asimismo, que estos problemas personales sean de relación y de conflicto de normas y reglas.

\section{Método}

\section{Participantes}

Los participantes en este estudio fueron 24 adolescentes residentes en un centro de acogida de Melilla, con edades comprendidas entre los 11 y los 18 años de los que una amplia mayoría (92\%) son varones.

Respecto a las circunstancias sociofamiliares de estos adolescentes, nos encontramos con que llevan un tiempo acogidos en este centro debido a encontrarse en situación de desamparo, siendo muchos de ellos menores extranjeros no acompañados (inmigrantes sin figura adulta que asuma su tutela).

\section{Instrumentos y procedimiento}

A todos los sujetos se le entrevistó a través de un cuestionario y de forma individualizada, garantizando el anonimato. Las entrevistas fueron llevadas a cabo por personal previamente entrenado, en horario de la mañana, durante el mes de agosto. Previamente a la entrevista, se citaba a un grupo reducido de menores (4) y se les explicaba la tarea que se les iba a solicitar (relatar la situación o problema que más le hubiese preocupado en los últimos 12 meses). A continuación, se asignaba cada menor a un entrevistador y comenzaba la entrevista, utilizándose los primeros momentos para presentarse y conseguir un clima relajado. A lo largo de la entrevista, se iba anotando la narración del menor. Posteriormente, los entrevistadores codificaban los problemas narrados según el Sistema de Codificación y Análisis Diferencial de los Problemas de los Adolescentes de Forns et al. (2004). Cada entrevista era codificada por dos de 
los entrevistadores que llegaban a un acuerdo sobre el código que debía asignarse. Con ello se pretendía disminuir o eliminar los problemas metodológicos asociados a la asignación de los códigos (diferencias interjueces).

\section{Análisis Estadístico}

Los datos obtenidos a través de la codificación de las entrevistas fueron tratados analizando la ocurrencia de cada categoría y convirtiendo, posteriormente, estas puntuaciones en porcentajes, tal como se realiza en el estudio de referencia citado, para lo que se utilizó un programa informático de cálculo (EXCEL).

\section{Resultados}

Los resultados muestran que los menores residentes en centros de acogida, presentan diferencias significativas en un importante número de variables, entre las que destacan la ocurrencia de problemas interpersonales $(70.83 \%)$ de relación $(41.67 \%)$ con compañeros $(33.33 \%)$ y que ocurren en el centro $(16.67 \%)$, respecto a los menores que viven con sus familias (véanse Tablas 1 y 2 ).

\section{TABLA 1}

Porcentajes de ocurrencia de los problemas según su naturaleza, contenido, participantes y contexto en los menores acogidos

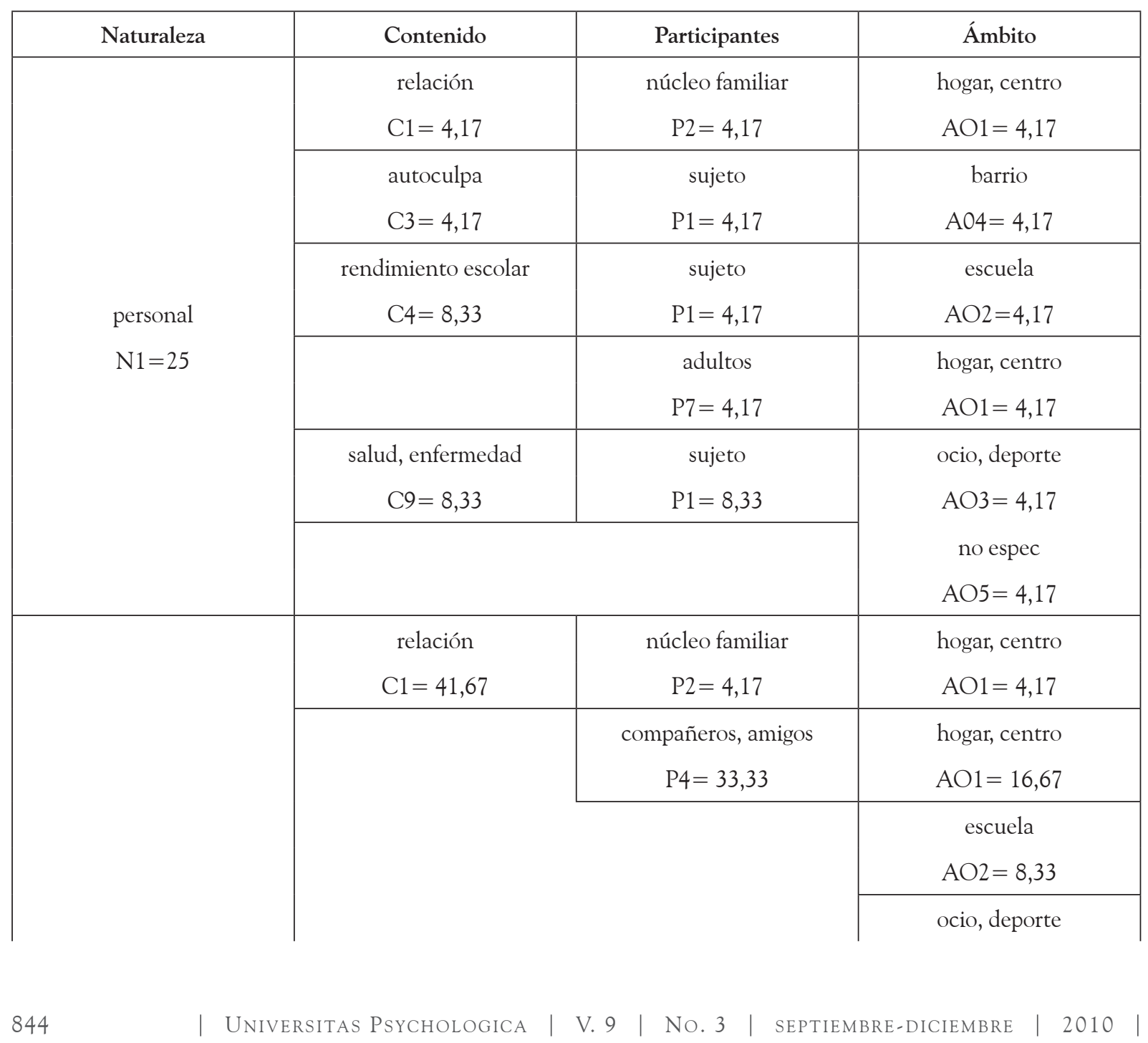




\begin{tabular}{|c|c|c|c|}
\hline Naturaleza & Contenido & Participantes & Ámbito \\
\hline \multirow{15}{*}{$\begin{array}{l}\text { interpersonal } \\
\qquad \mathrm{N} 2=70,83\end{array}$} & & & $\mathrm{AO} 3=8,33$ \\
\hline & & profesores & escuela \\
\hline & & $P 6=4,17$ & $\mathrm{AO} 2=4,17$ \\
\hline & divorcio. separación & pareja & ocio, deporte \\
\hline & $C 2=4,17$ & $\mathrm{P} 5=4,17$ & $\mathrm{AO} 3=4,17$ \\
\hline & conflicto con normas & compañeros, amigos & escuela \\
\hline & $\mathrm{C} 6=8,33$ & $\mathrm{P} 4=4,17$ & $\mathrm{AO} 2=4,17$ \\
\hline & & adultos & hogar, centro \\
\hline & & $\mathrm{P} 7=4,17$ & $\mathrm{AO} 1=4,17$ \\
\hline & deporte, ocio & compañeros, amigos & hogar, centro \\
\hline & $\mathrm{C} 8=16,67$ & $P 4=16,67$ & $\mathrm{AO} 1=4,17$ \\
\hline & & & ocio, deporte \\
\hline & & & $\mathrm{AO} 3=4,17$ \\
\hline & & & barrio \\
\hline & & & $\mathrm{AO} 4=8,33$ \\
\hline ajeno & conflicto con normas & compañeros, amigos & escuela \\
\hline $\mathrm{N} 3=4,14$ & $\mathrm{C} 6=4,14$ & $P 4=4,14$ & $\mathrm{AO} 2=4,14$ \\
\hline
\end{tabular}

Fuente: elaboración propia.

\section{TABLA 2}

Porcentajes de problemas en cada una de las categorías para los menores acogidos y los que viven con su familia (normalizados) y significación según la muestra

\begin{tabular}{|c|c|c|c|c|c|c|}
\hline Categorías del problema & & $\%$ acogidos & $\%$ normalzados & $z$ & & $P$ \\
\hline \multirow[t]{3}{*}{ Naturaleza } & Personal & 25,00 & 23,70 & & NS & \\
\hline & Interpersonal & 70,83 & 44,90 & 2.807 & & 0.005 \\
\hline & Ajeno & 4,17 & 27,70 & -4.265 & & 0.005 \\
\hline \multirow[t]{7}{*}{ Contenido } & Relación & 45,83 & 29,30 & 2.760 & & 0.005 \\
\hline & Divorcio & 4,17 & 2,70 & 3.291 & & 0.005 \\
\hline & Autoculpa & 4,17 & 5,60 & & NS & \\
\hline & Rendimiento escolar & 8,33 & 13,20 & & NS & \\
\hline & Cambios de hogar & 0,00 & 1,90 & -7.251 & & 0.005 \\
\hline & Conflictos normas & 12,50 & 8,80 & & NS & \\
\hline & Victimización & 0,00 & 3,10 & -6.029 & & 0.005 \\
\hline
\end{tabular}




\begin{tabular}{|c|c|c|c|c|c|c|}
\hline Categorías del problema & & $\%$ acogidos & $\%$ normalzados & Z & & $P$ \\
\hline & $\begin{array}{l}\text { Deporte, ocio. Vaca- } \\
\text { ciones }\end{array}$ & 16,67 & 0,60 & 238.678 & & 0.005 \\
\hline & $\begin{array}{l}\text { Salud, enfermedad, } \\
\text { accidentes }\end{array}$ & 8,33 & 15,80 & & NS & \\
\hline & Adicciones & 0,00 & 1,10 & -18.058 & & 0.005 \\
\hline & $\begin{array}{l}\text { Dinero, economía, } \\
\text { trabajo }\end{array}$ & 0,00 & 1,10 & -18.058 & & 0.005 \\
\hline & Muerte, suicidio & 0,00 & 11,50 & -5.176 & & 0.005 \\
\hline \multirow[t]{7}{*}{ Participantes } & Uso propio & 16,67 & 26,20 & & NS & \\
\hline & Núcleo familiar & 12,50 & 24,70 & & NS & \\
\hline & Familia extensa & 0,00 & 15,40 & -5.113 & & 0.005 \\
\hline & Compañeros, amigos & 58,33 & 20,20 & 9.097 & & 0.005 \\
\hline & Pareja & 4,17 & 4,70 & & NS & \\
\hline & Profesores & 4,17 & 2,20 & 5.761 & & 0.005 \\
\hline & Adultos & 4,17 & 1,60 & 12.123 & & 0.005 \\
\hline \multirow[t]{5}{*}{ Ámbitos } & Hogar & 37,50 & 40,2 & & NS & \\
\hline & Instituto & 25,00 & 22,2 & & NS & \\
\hline & Ocio & 20,83 & 3,7 & 24.038 & & 0.005 \\
\hline & Barrio & 12,50 & 3,7 & 12.892 & & 0.005 \\
\hline & Inespecífico & 4,17 & 30,2 & -4.322 & & 0.005 \\
\hline
\end{tabular}

Fuente: elaboración propia.

- Naturaleza del problema: la mayoría de los problemas expresados por los adolescentes son de naturaleza interpersonal (70.83\%), seguido de los de naturaleza personal (25.00\%).

- Contenido del problema: los problemas que poseen rasgos de relación son los que se perciben como más estresantes (45.83\%), seguidos por los problemas relacionados con el deporte, el ocio y las vacaciones que se presentan en el $16.67 \%$ de las ocasiones. También la conflictividad con normas y reglas ocupa un lugar importante dentro de los problemas percibidos (12.50\%).

- Participantes: respecto a los intervinientes en el conflicto, destacan los problemas relacionados con los compañeros o amigos (58.33\%).
- Ámbito de ocurrencia: parece ser que las situaciones más estresantes tienen lugar en el hogar, centro de acogida (37.50\%), seguido de los que ocurren en la escuela o instituto (25.00\%), y en lugares de ocio o deporte (20.83\%).

\section{Discusión y Conclusiones}

El objetivo de este trabajo era codificar los problemas percibidos por los menores acogidos en un centro por encontrarse en situación de desamparo, y comparar estos datos con los aportados por en el estudio de Forns sobre una muestra de adolescentes que vivían en el hogar familiar.

De la comparación de los resultados de ambas muestras (véase Tabla 2) se desprende que existen 
diferencias en el tipo de situación que afecta al menor. Así, respecto a la naturaleza del problema, si bien para ambas muestras son las relaciones interpersonales las más conflictivas, cuantitativamente se observa un mayor porcentaje en los adolescentes acogidos (más de un $70 \%$ frente a un $45 \%$ en los menores que viven en casa). Este resultado puede interpretarse aludiendo a un mayor tiempo y una mayor cantidad de interacciones entre los menores acogidos. Frente a ello, el bajo porcentaje que estos adolescentes presentan ante problemas ajenos $(4.17 \%)$, puede deberse a que los menores acogidos tienen un mayor número de problemas propios (dadas sus circunstancias sociofamiliares), quedando la preocupación por los problemas de los demás relegada a un segundo término. Otra posible explicación sería una falta o pobre capacidad empática, lo que sería objeto de otros estudios.

En cuanto al contenido de los problemas que más preocupa, ocurre algo similar a la naturaleza del problema, en ambas muestras son los problemas de relación, pero aquí de nuevo aparecen diferencias cuantitativas significando más del 45\% en acogidos y sólo un 29\% en menores "normalizados". La explicación puede residir en la importancia adaptativa que tienen las relaciones en un entorno de grupo, como es un centro de acogida de tamaño medio (cerca de 30 menores por pabellón). Acorde con esta idea, está el resultado obtenido en la categoría de participantes, en la que podemos observar que para los menores residentes son sus compañeros y amigos (58.33\%), y no el propio sujeto o la familia, como ocurre con los menores normalizados. La inexistencia de problemas relacionados con la familia extensa $(\mathrm{P} 3=0.00 \%)$ se debe a la nula o casi inexistente relación con ésta. Volviendo a la categoría de Contenido, ha de hacerse mención también del nivel de conflictividad que supone para los menores acogidos (16.67\%), frente al que presentan los normalizados (0.60\%). Esta diferencia puede deberse a una falta de habilidades sociales, como es la asertividad, de los menores acogidos, lo que se reflejaría en más conflictos de juego y ocio, en las que estas habilidades adquieren mayor importancia.
Por último, respecto al ámbito de ocurrencia, en el que más estrés se aprecia en los menores acogidos es el contexto de hogar-centro (37.50\%). Este resultado, no difiere en gran medida del encontrado en la muestra de menores normalizados (40.20\%).

Según los datos obtenidos, podemos concluir que las circunstancias que rodean al adolescente van a determinar, en gran parte, la percepción de sus problemas y la aparición de conflictos que son vividos en mayor o menor medida como estresantes. Así, el hecho de encontrarse en situación de desamparo (menores extranjeros no acompañados, menores abandonados, maltratados, sin familia, sin recursos o con padres fallecidos o encarcelados) y vivir en una residencia distinta a la familiar, es lo que distingue a las muestras utilizadas en ambos estudios (con la cautela sobre la interpretación de los datos que se precisa por el pequeño número de participantes en este trabajo), y es, al menos, una de las variables que determina los problemas que rodean al adolescente y la importancia que éste le da a los mismos.

\section{Referencias}

Brown, J. M., O’Keeffe, J., Sanders, S. H. \& Baker, B. (1986). Developmental changes in children's cognition to stressful and painful situations. Journal of Pediatric Psychology, 11, 343-357.

Castillo, G. (1999). El adolescente y sus retos. Madrid: Pirámide.

Coddington, R. D. (1972). The significance of life events as etiologic factors in the diseases of children. II. A study of a normal population. Journal of Psychosomatic Research, 16, 205-213.

Costa, M. \& Morales, J. M. (1998). ¿Por qué hay niños que cuando jóvenes llegan a comportarse violentamente? Claves para comprender el desarrollo de la violencia. Anuario de Psicología Jurídica, 8, 163-179.

Del Barrio, C. (1995). Los conflictos con los padres desde la perspectiva de los hijos. Infancia y Sociedad, 30, 133-143.

Espada, J. P., Méndez, X., Griffin, K. W. \& Botvin, G. J. (2003). Adolescencia: consumo de alcohol y otras drogas. Papeles del Psicólogo, 23, 9-17. 
Fernández-Millán, J. M. \& Buela-Casal, G. (2007). Padres desesperados con hijos adolescentes. Madrid: Pirámide.

Forns, M., Amador, J. A., Kirchner, T., Martorell, B., Zanini, D. \& Muro, P. (2004). Sistema de Codificación y Análisis Diferencial de los Problemas de los Adolescentes. Psicothema, 16, 646-653.

Funes, J. (2003). ¿Cómo trabajar con adolescentes sin empezar por considerarlos un problema? Papeles del Psicólogo, 23, 1-8.

Garaigordobil, M. (2000). Intervención psicológica con adolescentes. Madrid: Pirámide.

Gómez-Fraguela, J., Luengo-Martín, A., Romero-Triñanes, E., Villar-Torres, P. \& Sobral-Fernández, J. (2006). Estrategias de afrontamiento en el inicio de la adolescencia y su relación con el consumo de drogas y la conducta problemática. International Journal of Clinical and Health Psychology, 6, 581-597.

Hall, G. S. (1904). Adolescence: Its psychology and its relations to physiology, anthropology, sociology, sex, crime, religion, and education. New York: Appleton.

Kirchner, T. \& Forns, M. (2000). Autopercepción de "life events" como factores de riesgo y protección en adolescentes. Estudio diferencial. Revista de Psiquiatría. Facultad de Medicina de Barcelona, 27, 63-76.

Krauskopf, D. (1995). Dimensiones del desarrollo y la salud mental en la adolescencia. En Indicadores de Salud en la Adolescencia. San José, Costa Rica: O.P.S.

Maciá, D. (1994). Un adolescente en mi vida. Madrid: Pirámide.

Moos, R. (1993). Coping Responses Inventory Youth-form. Professional manual. Odessa, Florida: Psychological Assessment Resources.

Ochaíta, E. (1995). Desarrollo de las relaciones padre/ hijo. Infancia y Sociedad, 30, 207-228.

Urra, J. (1995). Adolescentes en conflicto. Madrid: Pirámide.

Urra, J. (2006). El Pequeño dictador. Madrid: La esfera de los libros.

Zavala, M. A., Valadez, M. D. \& Vargas, M. C. (2008). Emotional intelligence and social skills in adolescents with high social acceptance. Electronic Journal of Research in Educational Psychology, 6 (2), 319-338. 